ËESTÍ NSV TÉADUSítE AKADÉEMIA TOIMĖTISED, 31. KOIDË FOOSIKA * MATEMAATIKA. 1982, NR. 3

ИЗВЕСТИЯ АКАДЕМИИ НАУК ЭСТОНСКОП ССР. ТОМ 31 ФИЗИКА * МАТЕМАТИКА, 1982, № 3

удК 519.853 .4

Маэ УРИ

\title{
РЕШЕНИЕ МНОГОЭКСТРЕМАЛЬНОЙ ЗАДАЧИ РАЗМЕЩЕНИЯ МЕТОДОМ ВЕТВЕИ И ГРАНИЦ
}

\author{
(Представил А. Хумал)
}

\section{1. Постановка задачи размещения производства}

Рассмотрим задачу размещения производства однородного продукта с кусочно-линейной целевой функцией (назовем ее зад а че й А (ср. [ $\left.{ }^{1}\right]$, c. $52-54))$ : минимизировать функцию

$$
\sum_{i=1}^{m} \sum_{j=1}^{n} c_{i j} x_{i j}+\sum_{i=1}^{m} \sum_{k=1}^{m_{l}}\left(e_{i k} t_{i k}+d_{i, k-1} y_{i k}\right)
$$

при условиях

$$
\begin{gathered}
\sum_{i=1}^{m} x_{i j}=b_{j} \quad(j=\overline{1, n}), \\
\sum_{j=1}^{n} x_{i j}=\sum_{k=1}^{m_{t}}\left(a_{i, k-1} y_{i k}+t_{i k}\right) \quad(i=\overline{1, m}), \\
0 \leqslant t_{i k} \leqslant\left(a_{i k}-a_{i, k-1}\right) y_{i k} \quad\left(i=\overline{1, m} ; k=\overline{1, m_{i}}\right), \\
x_{i j} \geqslant 0, \quad y_{i k} \geqslant 0 \quad\left(i=\overline{1, m} ; j=\overline{1, n ; k}=\overline{1, m_{i}}\right), \\
\sum_{k=1}^{m_{f}} y_{i k} \leqslant 1 \quad(i=\overline{1, m}), \\
y_{\text {ik }}-\text { целые } \quad\left(i=\overline{1, m} ; k=\overline{1, m_{i}}\right) .
\end{gathered}
$$

Коэффициенты и переменные задачи А будем интерпретировать следующим образом: $x_{i j}$ - объем перевозок из пункта $i$ в пункт $j ; c_{i j}-$ удельные транспортные издержки, связанные с перевозкой единицы груза из пункта производства $i$ в пункт потребления $j ; e_{i k}-$ затраты на производство единицы продукта по $k$-му варианту в пункте $i ; t_{i k}-$ объем продукции, производимой по $k$-му варианту в пункте $i ; d_{i, k-1}-$ фиксированная доплата для достижения производственной мощности по $k$-му варианту в пункте $i ; a_{i, k-1}-$ минимальная производственная мощность пункта $i$, работающего по $k$-му варианту ( $a_{i m t}-$ максимальная мощность) $; b_{j}$ - потребность в продукции в пункте $j$.

Введем обозначение $\Delta a_{i k}=a_{i k}-a_{i, k-1}$ и предположим, что $d_{i k} \geqslant d_{i, k-1}+e_{i k} \Delta a_{i k}=h_{i k}, \quad d_{i 0}>0, \quad e_{i k} \geqslant e_{i, k+1}>0, \quad 0=a_{i 0}<a_{i 1}<\ldots<$ $<a_{i m_{i}}$ и

$$
h_{i k} / a_{i k}>h_{i, k+1} / a_{i, k+1} \quad\left(k=\overline{1, m_{i}-1}\right) .
$$


Кусочно-линейная разрывная функция затрат

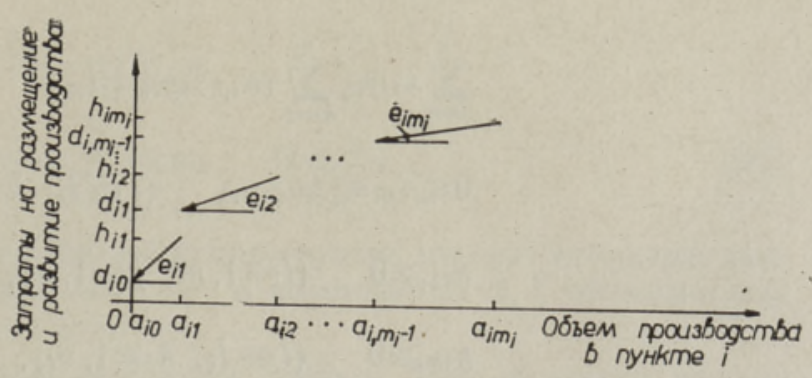

Спецификой исследуемой задачи размещения является нелинейная зависимость затрат на размещение и развитие производства от объема производства. В модели задачи нелинейность явно не выражается благодаря использованию целочисленных переменных $y_{i k}$. В целевой функции учитывается то обстоятельство, что с увеличением мощности предприятия фиксированные доплаты возрастают, но расходы на производство единицы продукта сокращаются. Функция затрат на размещение и развитие производства показана на рисунке.

Л. С. Лэсдон ([ $\left.{ }^{1}\right]$, с. 54) предлагает решать задачу такого типа методом разложения Бендерса: сначала фиксировать некоторые значения $y_{i k}$ и решать соответствующую линейную задачу, затем решать целочисленную задачу для определения новых значений $y k_{k}$. Дополнительные ограничения в целочисленной задаче определяются из оптимального решения задачи, двойственной к линейной. Б. И. Алейников $\left[{ }^{2,3}\right]$ расматривает эту задачу в несколько других постановках, в том числе и в сетевой. Он предлагает для нахождения локальных оптимумов метод случайного поиска и метод линеаризации.

Для решения задачи А мы будем исследовать полученные методом типа ветвей и границ подзадачи, в которых часть $y_{\text {i九 }}$ фиксирована, т. е. принята равной нулю или единице (кратко $y_{i k}=1$ фикс.). Введем обозначения

$$
\begin{aligned}
& I_{1}=\left\{i \mid \exists k_{i} \in\left\{1,2, \ldots, m_{i}\right\}: y_{i k_{i}}=1 \text { фикс. }\right\} \\
& I_{c}=\{1,2, \ldots, m\} \backslash I_{1} ; \\
& v_{i}=\max \left\{k \mid y_{i k}-\text { не фиксированы }\right\}, i \in I_{c} ; \\
& X=\left(x_{i j}\right), Y=\left(y_{i k}\right), T=\left(t_{i k}\right) \quad\left(i=\overline{1, m}, j=\overline{1, n}, k=\overline{1, v_{i}}\right) .
\end{aligned}
$$

Целевую функцию подзадачи получим из (1), учитывая, что при $i \in I_{1} \quad y_{i k_{t}}=1$ и $t_{i k}=y_{i k}=0 \quad\left(k \neq k_{i}\right)$, а из (3) следует $t_{i k_{t}}=$ $=\sum_{j=1}^{n} x_{i j}-a_{i, k t-1}$. Таким образом, можем сформулировать подзадачу (назовем ее зад а ч ей АФ): минимизировать функцию

$$
\begin{aligned}
& z(X, Y, T) \equiv \sum_{i \in I_{c}} \sum_{j=1}^{n} c_{i j} x_{i j}+\sum_{i \in I_{c}} \sum_{k=1}^{v_{t}}\left(e_{i k} t_{i k}+d_{i, k-1} y_{i k}\right)+ \\
& +\sum_{i \in I_{1}} \sum_{j=1}^{n}\left(c_{i j}+e_{i k_{i}}\right) x_{i j}+\sum_{i \in I_{1}}\left(d_{i, k_{i}-1}-e_{i k_{i}} a_{i, k_{i}-1}\right)
\end{aligned}
$$

при условиях

$$
\begin{aligned}
& \sum_{i=1}^{m} x_{i j}=b_{j} \quad(j=\overrightarrow{1, n)}, \\
& a_{i, k_{t}-1} \leqslant \sum_{j=1}^{n} x_{i j} \leqslant a_{i k_{t}} \quad\left(i \in I_{1}\right),
\end{aligned}
$$




$$
\begin{aligned}
& \sum_{j=1}^{n} x_{i j} \leqslant \sum_{k=1}^{v_{t}}\left(a_{i, k-1} y_{i k}+t_{i k}\right) \quad\left(i \in I_{c}\right), \\
& 0 \leqslant t_{i k} \leqslant \Delta a_{i k} y_{i k} \quad\left(i \in I_{c} ; k=\overline{1, v_{i}}\right), \\
& x_{i j} \geqslant 0 \quad(i=\overline{1, m} ; j=\overline{1, n}), \\
& y_{i k} \geqslant 0 \quad\left(i \in I_{c} ; k=\overline{1, v_{i}}\right), \\
& \sum_{h=1}^{v_{t}} y_{i k} \leqslant 1 \quad(i=\overline{1, m}), \\
& y_{i k} \text { - целые } \quad\left(i \in I_{c} ; k=\overline{1, v_{i}}\right) .
\end{aligned}
$$

Соответствующую АФ непрерывную задачу (9)-(16) будем называть з а д а ч ей БФ. В случае $I_{1}=\varnothing$ и $v_{i}=m_{i}(i=\overline{1, m})$ задача АФ совпадает с задачей $\mathrm{A}$.

\section{2. Свойства оптимальных решений задачи БФ}

Те орем а 1. Если $\left(X^{0}, Y^{0}, T^{0}\right)$ есть оптимальное решение непрерывной задачи БФ, то

$$
t_{i k}^{0}=\Delta a_{i k} y_{i k}^{0} \quad \text { при } \quad i \in I_{c}, \quad k=\overline{1, v_{i}} .
$$

Доказательство. а) Если $y_{i h}{ }^{0}=0$, то из (13) следует $t_{i k}{ }^{0}=0$ $\left(i \in I_{c}\right)$. б) Предположим, что существует $y_{r s}{ }^{0}>0$, но $t_{r s}{ }^{0}<\Delta a_{r s} y_{r s}{ }^{0}$ $\left(r \in I_{c}\right)$. Выберем новое допустимое решение $\left(X^{0}, Y^{0}-\Delta Y^{0}, T^{0}+\Delta T^{0}\right)$, получаемое из вектора $\left(X^{0}, Y^{0}, T^{0}\right)$ уменьшением $y_{r s}{ }^{0}$ на $\Delta y_{r s}{ }^{0}>0$ и увеличением $t_{r s}{ }^{0}$ на $\Delta t_{r s}{ }^{0}>0$, так что

$$
t_{r s}^{0}+\Delta t_{r s}^{0}=\Delta a_{r s}\left(y_{r s}^{0}-\Delta y_{r s}^{0}\right),
$$

Из допустимости обоих векторов $\left(X^{0}, Y^{0}, T^{0}\right)$ и $\left(X^{0}, Y^{0}-\Delta Y^{0}, T^{0}+\Delta T^{0}\right)$ вытекает соответственно $\quad \sum_{j=1}^{n} x_{r j}^{0}=\sum_{k=1}^{v_{t}}\left(a_{r, k-1} y_{r k}^{0}+t_{r k}^{0}\right) \quad$ и $\quad \sum_{j=1}^{n} x_{r j}^{0}=$ $=\sum_{k=1, k \neq 8}^{v_{t}}\left(a_{r, k-1} \stackrel{0}{y_{r k}}+t_{r k}^{0}\right)+a_{r, s-1}\left(y_{r s}^{0}-\Delta y_{r s}^{0}\right)+t_{r s}^{0}+\Delta t_{r s}^{0}$, т. e. $\Delta t_{r s}^{0}=a_{r, s-1} \Delta y_{r s}^{0}$. Из (19) следует, что $\Delta y_{r s}^{0}=\left(\Delta a_{r s} y_{r s}^{0}-t_{r s}^{0}\right) / a_{r s}>0$. Учитывая, что $e_{i k}<h_{i k} / a_{i k}<h_{i, k-1} / a_{i, k-1} \leqslant d_{i, k-1} / a_{i, k-1}$, $\quad$ т. е. $\quad e_{i k}<d_{i, k-1} / a_{i, k-1}$, вычислим разницу между значениями целевой функции при рассматриваемых векторах:

$$
z\left(X^{0}, Y^{0}, T^{0}\right)-z\left(X^{0}, Y^{0}-\Delta Y^{0}, T+\Delta T^{0}\right)=\left(d_{r, s-1}-e_{r s} a_{r, s-1}\right) \Delta y_{r s}^{0}>0 .
$$

Результат противоречит предположению об оптимальности $\left(X^{0}, Y^{0}, T^{0}\right)$. Следовательно, и при $y_{i k}{ }^{0}>0$ имеет место (18). Теорема доказана.

Из предыдущего вытекает, что непрерывная задача БФ эквивалентна зад ач е ВФ: минимизировать функцию

$$
\begin{gathered}
z(X, Y) \equiv \sum_{i \in I_{c}} \sum_{j=1}^{n} c_{i j} x_{i j}+\sum_{i \in I_{c}} \sum_{k=1}^{v_{t}} h_{i k} y_{i k}+\sum_{i \in I_{1}} \sum_{j=1}^{n}\left(c_{i j}+e_{i k_{i}}\right) x_{i j}+ \\
+\sum_{i \in I_{t}}\left(d_{i, k_{i}-1}-e_{i k_{i}} a_{i, k_{i}-1}\right)
\end{gathered}
$$


при условиях (10), (11), (14)-(16) и

$$
\sum_{j=1}^{n} x_{i j}=\sum_{k=1}^{v_{l}} a_{i k} y_{i k} \quad\left(i \in I_{c}\right) .
$$

Задачи БФ и ВФ эквивалентны в том смысле, что оптимальные значения их целевых функций совпадают и компоненты $X, Y$ оптимальных решений имеют одинаковые значения.

Теорема 2. Оптимальным решением задачи ВФ является вектор $\left(X^{0}, Y^{0}\right), \quad к о г д a \quad y_{i v_{i}}^{0}=\left(\sum_{j=1}^{n} x_{i j}^{0}\right) / a_{i v_{i}}, \quad y_{i k}^{0}=0 \quad\left(k \neq v_{i}\right) \quad$ nрu $i \in I_{c} \quad u$ $y_{i k_{i}}^{0}=1, y_{i k}^{0}=0 \quad\left(k \neq k_{i}\right) \quad$ при $i \in I_{1}$, a $X^{0}$ есть оптимальное решение задач и ТФ: минимизировать функцию

$$
\begin{aligned}
z(X) \equiv \sum_{i \in I_{c}} \sum_{j=1}^{n}\left(c_{i j}+\frac{h_{i v_{i}}}{a_{i v_{i}}}\right) x_{i j}+\sum_{i \in I_{1}} \sum_{j=1}^{n}\left(c_{i j}+e_{i k_{i}}\right) x_{i j}+ \\
+\sum_{i \in I_{1}}\left(d_{i, k_{i}-1}-e_{i k_{i}} a_{i, k_{i}-1}\right)
\end{aligned}
$$

при условиях (10), (11) $u$

$$
\begin{gathered}
\sum_{j=1}^{n} x_{i j} \leqslant a_{i v_{i}} \quad\left(i \in I_{c}\right), \\
x_{i j} \geqslant 0 \quad(i=\overline{1, m} ; j=\overline{1, n)} .
\end{gathered}
$$

Доказ а тельство. Вначале покажем, что при $i \in I_{c}$ в оптимальном решении $\left(X^{0}, Y^{0}\right)$ задачи ВФ $y_{i v_{l}}^{0}>0$ и $y_{i k}^{0}=0 \quad\left(k \neq v_{i}\right)$. Предположим, что $\exists u\left(u \in I_{c}\right)$, при котором $y_{u k_{1}}^{0}>0, y_{u k_{2}}^{0}>0, \cdots, y_{u k_{p}}^{0}>0$, причем либо $p=1$ и $k_{p}<v_{u}$, либо $p>1$ и $k_{p} \leqslant v_{u}$, а остальные $y_{u k}{ }^{0}=0$. Оптимальное решение $\left(X^{0}, Y^{0}\right)$ удовлетворяет условиям (21), (15) и (16), т. е.

$$
\sum_{j=1}^{n} x_{u j}^{0}=\sum_{r=1}^{p} a_{u k_{r}} y_{u k_{r}}^{0} \leqslant a_{u v_{u}} \quad \text { и } \quad \sum_{r=1}^{p} y_{u k_{r}}^{0} \leqslant 1
$$

Рассмотрим вектор $\left(X^{0}, Y^{\prime}\right)$, когда $y^{\prime} u v_{u}=\sum_{r=1}^{p}\left(a_{u k_{r}} / a_{u v_{u}}\right) y_{u k_{r}}^{0}, y^{\prime} u k=0$ $\left(k \neq v_{u}\right), \quad y_{i k}^{\prime}=y_{i k}^{0} \quad(i \neq u) . \quad$ Он является допустимым, так как $\sum_{k=1}^{v_{u}} a_{u k} y^{\prime}{ }_{u k}=a_{u v_{u}} y_{u v_{u}}^{\prime}=\sum_{r=1}^{p} a_{u k_{r}} y_{u k_{r}}^{0}=\sum_{j=1}^{n} x_{u j}^{0} \quad$ и $\quad y^{\prime}{ }_{u v_{u}}<\sum_{r=1}^{p} y_{u k_{r}}^{0} \leqslant 1 . \quad$ Раз. ница между $u$-ми членами целевой функции при $\left(X^{0}, Y^{0}\right)$ и $\left(X^{0}, Y^{\prime}\right)$ имеет вид

$$
\begin{gathered}
\sum_{r=1}^{p} h_{u k_{r}} y_{u k_{r}}^{0}-h_{u v_{u}} y_{u v_{u}}^{\prime}=\sum_{r=1}^{p}\left(h_{u k_{r}}-\frac{a_{u k_{r}}}{a_{u v_{u}}}\right) y_{u k_{r}}^{0}= \\
=\sum_{r=1}^{p} a_{u k_{r}}\left(\frac{h_{u k_{r}}}{a_{u k_{r}}}-\frac{h_{u v_{u}}}{a_{u v_{u}}}\right) y_{u k_{r}}^{0}
\end{gathered}
$$

и является положительной в силу предположения (8). Но это противоречит предположению об оптимальности $\left(X^{0}, Y^{0}\right)$; следовательно, в 
$\left(X^{0}, Y^{0}\right)$ положительна лишь $y_{i v_{i}}^{\text {a }}\left(i \in I_{c}\right)$. Из (21) следует, что $y_{i v_{i}}^{0}=\left(\sum_{j=1}^{n} x_{i j}^{0}\right) / a_{i v_{i}}$.

Проверим на оптимальность допустимое решение $\left(X^{1}, Y^{1}\right)$, когда $X^{1} \neq X^{0}, y_{i v_{i}}^{1}=\left(\sum_{j=1}^{n} x_{i j}^{1}\right) / a_{i v_{i}}, y_{i k}^{1}=0\left(k \neq v_{i}\right) \quad$ при $i \in I_{c}$.

Получим

$$
\begin{gathered}
z\left(X^{1}, Y^{1}\right)=\sum_{i \in I_{c}} \sum_{j=1}^{n}\left(c_{i j}+\frac{h_{i v_{i}}}{a_{i v_{i}}}\right) x_{i j}^{1}+\sum_{i \in I_{1}} \sum_{j=1}^{n}\left(c_{i j}+e_{i k_{i}}\right) x_{i j}^{1}+ \\
+\sum_{i \in I_{1}}\left(d_{i, k_{i}-1}-e_{i k_{i}} a_{i, k_{i}-1}\right) \geqslant z\left(X^{0}\right)=z\left(X^{0}, Y^{0}\right) .
\end{gathered}
$$

Теорема доказана.

Т еор ем а 3. Минимальное значение целевой функции задачи ТФ является нижней границей минимального значения целевой функции задачи АФ.

Доказательство. Оптимальное решение задачи АФ является допустимым (но не обязательно оптимальным) для непрерывной задачи БФ, где от величин $y_{i k}\left(i \in I_{c}\right)$ целочисленности не требуется. Оптимальные значения целевых функций задач БФ, ВФ и ТФ совпадают. Теорема доказана.

Соответствующий оптимальному решению $\left(X^{0}, Y^{0}\right)$ задачи ВФ вектор $\left(X^{0}, Y^{\prime \prime}, T^{\prime \prime}\right)$, при котором $y_{i p_{i}}^{\prime \prime}=1, t_{i p_{i}}^{\prime \prime}=\sum_{j=1}^{n} x_{i j}^{0}-a_{i, p_{i}-1}, y_{i k}^{\prime \prime}=t_{i k}^{\prime \prime}=0$ $\left(k \neq p_{i}, \quad\right.$ а $p_{i}=v_{i} \quad$ при $\quad i \in I_{c}, \quad p_{i}=k_{i}$ при $\left.i \in I_{1}\right)$, может не быть допустимым решением задачи АФ, так как $t_{i v t}{ }^{\prime \prime}<0$ в случае $v_{i} \neq r_{i}=\min \left\{k \mid \sum_{j=1}^{n} x_{i j} \leqslant a_{i k}\right\} . \quad$ Из допустимого решения $\left(X^{0}, Y^{1}\right)$ задачи ВФ, когда $y_{i r_{i}}^{1}=\left(\sum_{j=1}^{n} x_{i j}^{0}\right) / a_{i r_{i}}, y_{i k}^{1}=0\left(k \neq r_{i}\right) \quad$ при $i \in I_{c}$, получим для задачи АФ допустимое решение $\left(X^{0}, Y^{\prime}, T^{\prime}\right)$, при котором $y_{i q_{i}}^{\prime}=1, t^{\prime}{ }_{i q_{i}}=\sum_{j=1}^{n} x_{i j}^{0}-a_{i, q_{i}-1}, y_{i k}^{\prime}=t_{i k}^{\prime}=0 \quad\left(k \neq q_{i}\right)$ и $q_{i}=r_{i}$ при $i \in I_{c}$, a $q_{i}=k_{i}$ при $i \in I_{1}$.

\section{3. Метод ветвей и границ для решения задачи $\mathrm{A}$}

Реализуем идею метода ветвей и границ (см., напр., $\left[{ }^{4}\right]$, с. $267-274$; $\left[{ }^{5}\right]$, с. $213-218 ;\left[{ }^{6}\right]$, с. $\left.161-167\right)$ для решения задачи А. Предлагаемый метод опирается на доказанные в разделе 2 теоремы и заключается в решении ряда подзадач типа ТФ - транспортных задач с дополнительными ограничениями на значения $\sum_{j=1}^{n} x_{i j}$. В соответствующей транспортной таблице подзадачи имеется не более $n \times m$ клеток. Задачу ТФ можно решить, например, модификацией метода потенциалов или метода разрешающих слагаемых.

Сначала решим задачу ТФ, где $I_{1}=\varnothing$ и $v_{i}=m_{i}(i=\overline{1, m})$. Соответствующий оптимальному плану $X^{0}$ задачи ТФ вектор $\left(X^{0}, Y^{\prime}, T^{\prime}\right)$, при котором $y_{\text {ir }_{i}}^{\prime}=1, \quad t_{i r_{i}}^{\prime}=\sum_{j=1}^{n} x_{i j}^{0}-a_{i, r_{i}-1}, \quad y_{i k}^{\prime}=t_{i k}^{\prime}=0 \quad\left(k \neq r_{i}, \quad r_{i}=\right.$ 
$\left.=\min \left\{k \mid \sum_{j=1}^{n} x_{i j}^{0} \leqslant a_{i k}\right\}\right)$, является рекордным решением задачи $\mathrm{A}$, а $z\left(X^{0}, Y^{\prime}, T^{j=1}\right.$ - рекордом. Если в оптимальном плане $\left(X^{0}, Y^{0}\right)$ задачи ВФ (см. теорему 2) $Y^{0}$ целочислен, то соответствующий ему вектор $\left(X^{0}, Y^{\prime}, T^{\prime}\right)$ будет оптимальным решением задачи А. Если же в оптимальном плане $\left(X^{0}, Y^{0}\right)$ задачн ВФ существует $y_{u v_{u}}^{0} \neq 1$, то проведем ветвление, добавляя к ограничениям задачи ВФ требования $y_{u v_{u}}=1$ и $y_{u v_{u}}=0$.

Какой индекс $i$ предпочесть при ветвлении, можно решить, руководствуясь, например, одним из следующих правил:

П1. Выбрать $i: \quad y_{i v_{i}}=\left(\sum_{j=1}^{n} x_{i j}^{0}\right) / a_{i v_{i}} \neq 1 \quad\left(i \in I_{c}\right)$, при котором значение $F_{i}=h_{i v t}+d_{i, v_{t}-1}$ наименьшее.

П2. Выбрать $i: y_{i v t} \neq 1 \quad\left(i \in I_{c}\right)$, при котором значение $M_{i}=$ $=\frac{1}{v_{i}} \sum_{k=1}^{v_{t}} h_{i k}$ наименьшее (аналогично $\left[{ }^{7}\right]$, с. 41 ).

Уменьшить число перебираемых вершин, а следовательно, и исключнть из рассмотрения подзадачи, не имеюшие допустимых решений или у которых значение целевой функции не меньше рекорда $f^{*}$ (рекордом $f^{*}$ назовем значение целевой функции задачи А при наилучшем, уже найденном допустимом решении), помогут приводимые ниже тесты.

T1 (тест неразрешимости): выполняется ли одно из условий

$$
\sum_{j=1}^{n} b_{j}>\sum_{i \in I_{1}} a_{i k_{i}}+\sum_{i \in I_{c}} a_{i v_{i}} \text { или } \sum_{j=1}^{n} b_{j}<\sum_{i \in I_{1}} a_{i, k_{i}-1} \text { ? }
$$

Т2 (сравнение оценки целевой функции с рекордом): выполняется ли условие $z_{-} \geqslant f^{*}$, когда

$$
\begin{aligned}
& z=\sum_{s=1}^{p} \sum_{j=1}^{n} g_{i_{s}(j), j} r_{i_{s}(j), j}+\sum_{i_{s}(j) \in I_{t}}\left(d_{i_{s}(j), k_{i}(j)}-1-e_{i_{s}(j), k_{i}(j)} a_{i_{s}(j), k_{i}(j)}-1\right), \\
& g_{i_{s}(j), j}=\min \left\{\min _{\substack{i \in I_{1} \\
i \neq i_{1}(j), \ldots, i_{s-1}(j)}}\left(c_{i j}+e_{i h_{i}}\right), \min _{\substack{i \in I_{\mathrm{c}} \\
i \neq i_{1}(j), \ldots, i_{v-1}(j)}}\left(c_{i j}+\frac{h_{i v_{i}}}{a_{i v_{i}}}\right)\right\},- \\
& r_{i_{0}(j), j}=0, r_{i_{s}(j), j}=\min \left\{b_{j}-\sum_{u=1}^{s-1} r_{i_{u}(j), j}, a_{i_{z}(j), q}\right\} \quad(j=\overline{1, n}, s=\overline{1, p}), \\
& p=\min \left\{u \mid u \geqslant 1, \quad r_{i_{u}(j), j}=0, j=\overline{1, n}\right\}, q=\left\{\begin{array}{l}
k_{i_{s}(j)} \text { при } i_{s}(j) \in I_{1}, \\
v_{i_{s}(j)} \text { при } i_{s}(j) \in I_{c} .
\end{array}\right.
\end{aligned}
$$

Т3 (сравнение значения целевой функции с рекордом): выполняется ли условие $z\left(X^{0}\right) \geqslant f^{*}$ ?

Тест Т4 проверяет, является ли вектор $\left(X^{0}, Y^{\prime}, T^{\prime}\right)$, соответствуюший оптимальному решению $X^{0}$ подзадачи ТФ, рекордным решением исходной задачи А.

T4: выполняется ли условие $z\left(X^{0}, Y^{\prime}, T^{\prime}\right)<f^{*}$ ?

Тест Т5 проверяет, является ли соответствующий оптимальному решению $X^{0}$ вектор $Y$ целочисленным.

Т5: выполняется ли условие $y_{i v_{i}}=\left(\sum_{j=1}^{n} x_{i j}^{0}\right) / a_{i v_{i}}=1 \quad \forall i \in I_{c}$ ? 
Изложим теперь алгоритм нахождения глобального оптимума задачи А. На каждой итерации известен рекорд $f^{*}$ и известен список задач-кандидатов $C$, где в процессе решения сохраняются подзадачи с нижними оценками $z_{-}<f^{*}$. Учитываем, что рассматриваемая задачакандидат получается из задачи ТФ $=\{(22),(10),(11),(23),(24)\}$ конкретизацией множеств $I_{1}, I_{c}$ и индексов $k_{i}\left(i \in I_{1}\right)$ и $v_{i}\left(i \in I_{c}\right)$, т. е. заменой их множествами $I_{1}^{l}, I_{c}^{l}$ и индексами $k_{i}^{l}, v_{i}^{l}$, где $l$ - номер кандидата.

Ш a r 0. Присваиваем $f^{*}:=\infty, C=\varnothing, I_{1}{ }^{1}=\varnothing, I_{c}{ }^{1}=\{1, \ldots, m\}$, $v_{i}{ }^{1}=m_{i}, z_{-}{ }^{1}=-\infty$. Проверяем задачу ТФ тестом Т1, при его выполнении переходим к шагу 1; в противном случае присванваем задаче ТФ номер 1 и включаем ее в список $C$.

Ш а г 1. Проверяем, пуст ли список $C$ ? Если $C=\varnothing$, то заканчиваем вычисления. В случае $f^{*}=\infty$ задача $\mathrm{A}$ не имеет допустимых решений; в противном случае рекордное решение $\left(X^{0}, Y^{\prime}, T^{\prime}\right)$ является точкой глобального минимума исходной задачи А. Если $C \neq \varnothing$, то выбираем, исключая, из списка $C$ в качестве очередного кандидата задачу с наименьшей оценкой $z_{-}$.

Ш а г 2. Решаем выбранную задачу. Проверяем оптимальный план тестом Т3, при его выполнении переходим к шагу 1.

Шаг 3. Проверяем оптимальный план тестом Т4, при его выполнении пересчитываем рекорд $f^{*}:=z\left(X^{0}, Y^{\prime}, T^{\prime}\right)$ и переходим к шагу 4 , иначе к шагу 5.

Шаг 4. Проверяем задачи из списка $C$ тестом Т2. Те из задач, при которых тест выполняется, исключаем из списка $C$.

Ш а г 5. Проверяем оптимальный план $X^{0}$ тестом $T 5$, при его выполнении переходим к шагу 1 , иначе - к шагу 6.

Шаг 6. Пусть номер рассматриваемого кандидата будет $l$. Проведем ветвление задачи $l$ по индексу $u$, выбранному по правилу П 1 , на две подзадачи с соответствующими множествами $I_{1}^{r+1}=I_{1}^{l} \bigcup\{u\}, I_{c}^{r+1}=I_{c}^{l} \backslash\{u\}$; $k_{u}^{r+1}=v_{u}^{l}$ и $I_{1}^{r+2}=I_{1}^{l}, I^{r+2}=I_{c}^{l} ; v_{u}^{r+2}=v_{u}^{!}-1, \quad$ где $r-$ наибольший номер уже генерированных подзадач. Вычисляем оценки $z_{-}^{r+1}$ и $z_{-}^{r+2}$ по формуле (25). Проверяем задачи с номерами $r+1$ и $r+2$ тестами Т1 и Т2. Задачи, при которых тесты не выполняются, включаем в список $C$. Переходим к шагу 1 .

\section{Л И ТЕР А Т У Р А}

1. Лэсдон Л. С.. Оптимизация больших систем, М., «Наука», 1975.

2. Алейников Б. И., Экономика и матем. методы, 2 , № 2, 272-282 (1966).

3. Алей ни ков Б. И.,, Мовшов и ч С. Г., В кн.: Исследования по математическому программированию, М., «Наука», 1968, с. $198-221$.

4. В а г е р Г., Основы нсследования операцин, т. 2, М., «Мир», 1973.

5. Корбут А. А., Финкельштей Н Ю. Ю., Дискретное программирование, М., «Наука», 1969.

6. Сергиенко И. В., Л ебедева Т. Т., Рощин В. А., Приближенные методы решения дискретных задач оптимизации, Киев, «Наукова думка», 1980.

7. Gauthier, J.-M., Ribiere, G., Math. Progr., 12, № 1, 26-47 (1977).

Тартуский государственный университет

Поступила в редакцию $20 / \mathrm{I} 1982$ 


\section{PALJUEKSTREMAALSE PAIGUTUSULESANDE LAHENDAMINE HARUDE JA TOKETE MEETODIL}

On vaadeldud tükiti-lineaarse sihifunktsiooniga paigutusülesannet (1)-(7), mille lahendamiseks on välja töötatud harude ja tõkete meetodi tüüpi algoritm. Lahenduse käigus on vaatluse all alamülesanded, milles csa $y_{i k}$ on fikseeritud, s. o. vōetud võrdseks nulli vōi ühega. Algoritm põhineb nimetatud alamülesandele vastava pideva planeerimisülesande $(9)-(16)$ optimaalsete lahendite teatud omadustel.

\section{Mae URI}

\section{SOLVING THE MULTIEXTREMAL LOCATION PROBLEM WITH THE BRANCH-AND-BOUND METHOD}

In this paper the location problem (1) - (7) with piecewise linear objective function (1) and constraints $(2)-(7)$ is discussed. An algorithm basing on the idea of branch-andbound method for 'solving this problem is presented. During the solving process subproblems are under discussion, in which part of variables $y_{i k}$ are fixed to zero or one. The algorithm is based on the qualities of the optimal solution of continuous problem (9)-(16) corresponding to the subnroblem (9)-(17). Since in the optimal solution $\left(X^{0}, Y^{0}, T^{0}\right)$ of the problem (9) - (16) between the components of $T^{0}$ and $X^{0}$ equality (18) is valid, then the problem $(9)-(16)$ is equivalent to the problem $\{(20),(10),(11)$, (14) - (16), (21)\}. The solution of the last problem is cancelled to solving a transportation problem with an additional condition on $\sum_{j=1}^{n} x_{i j}$. The choice of a branching variable is made by rules $\Pi 1$ and $\Pi 2$. In order to reduce the quantity of subproblems, some tests are used: the test of unsolvability.(T1), the test of comparison of the estimation of objective function with the record (T2) and the test of comparison of the minimum value of objective function with the record (T3). Correction of the record is made by the test $\mathrm{T} 4$ and the branch is terminated by the test.T5. 